\title{
Synthesis of $N^{4}$-aryl-p-D-glucopyranosylcytosines: a methodology study
}

\author{
Michael Mamais a,b, Virginia Kouloumoundra ${ }^{a}$, Eugenia Smyrli ${ }^{\text {a }}$, Paschalis Grammatopoulos ${ }^{\text {a }}$, \\ Evangelia D. Chrysina ${ }^{\mathrm{b}}$, Thanasis Gimisis ${ }^{\mathrm{a}, *}$ \\ a Department of Chemistry, National and Kapodistrian University of Athens, Panepistimiopolis, GR-15771 Athens, Greece \\ ${ }^{\mathrm{b}}$ Institute of Biology, Medicinal Chemistry and Biotechnology, National Hellenic Research Foundation, Athens GR-11635, Greece
}

\section{A R T I C L E I N F O}

\section{Article history:}

Received 11 March 2015

Revised 28 July 2015

Accepted 17 August 2015

Available online 20 August 2015

\section{Keywords:}

Substitution reaction

Arylamines

Triazoles

$N^{4}$-Aryl- $\beta$-D-glucopyranosylcytosines

Microwaves

\begin{abstract}
A B S T R A C T
A number of leaving groups, including arylsulfonates, triazoles, 3-nitrotriazoles, and tetrazoles, have been studied for the substitution reaction by aryl and alkyl amines at the 4-position of $\beta$-D-glucopyranosyluracils. Examination of the stability, ease of purification and reactivity in the substitution reaction led to a number of optimized conditions with the most convenient involving substitution of triazole derivatives under microwave conditions in the presence of silica gel. Under these conditions, a number of $N^{4}$-aryl-substituted $\beta$-D-glucopyranosylcytosines were prepared as potential inhibitors of glycogen phosphorylase, a molecular target for type-2 diabetes mellitus.
\end{abstract}

(c) 2015 Elsevier Ltd. All rights reserved.

\section{Introduction}

Arylsulfonyltriazoles and tetrazoles were utilized during the development of the phosphotriester method for oligonucleotide synthesis, in order to improve the mildness and efficiency of the coupling step. However, it soon became apparent that these reagents also reacted with the unprotected carbonyl groups at $O^{4}$ of uridine and $O^{6}$ of guanosine, furnishing 4-arylsulfonate, 4-triazole and 4-tetrazole derivatives as side products, thus necessitating the protection of these functional groups. ${ }^{1}$ The reactivity of 4-(1,2,4-triazolyl)- and 4-(3-nitro-1,2,4-triazolyl)-pyrimidin-2one derivatives and their substitution by oxygen and nitrogen nucleophiles was first studied in detail by Reese, ${ }^{2}$ who showed that these intermediates could be viable precursors for alkylamine and simple arylamine derivatives. In recent years, this methodology has been extensively used for converting uridine to cytidine ${ }^{3-5}$ as well as other $\mathrm{N}^{4}$-alkyl substituted nucleosides. ${ }^{6-9}$

Glycogen phosphorylase (GP) is a validated molecular target for the development of antidiabetic drugs. ${ }^{10}$ In the course of our studies toward the design and synthesis of potent inhibitors of GP, ${ }^{11,12}$ we required an efficient and general methodology for substitution of the $\mathrm{O}^{4}$ position of $\beta$-D-glucopyranosyluracil by various arylamines. This interest required us to test the reactivity of a wide

\footnotetext{
* Corresponding author. Tel.: +30 210727 4477; fax: +30 2107274761 .

E-mail address: gimisis@chem.uoa.gr (T. Gimisis).
}

range of electron rich and poor arylamines for the synthesis of $N^{4}$-aryl- $\beta$-D-glucopyranosylcytosines. We report herein the results of a methodology study that was performed in order to determine the best activating group, additive and reaction conditions for the above reaction, which was amenable to a large variety of aryl and alkyl amines.

\section{Results and discussion}

Initially, we attempted to apply the conditions developed by Vorbrüggen and coworkers, ${ }^{13}$ utilizing $O^{4}$-TMS as the leaving group (1, Fig. 1). However, reaction of trimethylsilylated 2,3,4,6-tetra-Oacetyl- $\beta$-D-glucopyranose with 2 -aminofluorene (2AF) did not lead to the formation of any product. It was noted that only primary and secondary alkylamines were utilized by Vorbrüggen whereas
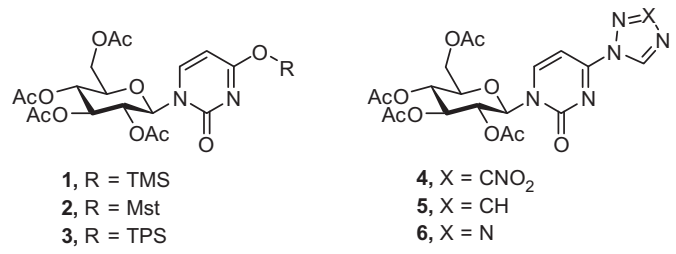

Figure 1. $O^{4}$-activated $\beta$-D-glycopyranosylpyrimid-2-ones studied in this work. (TMS: trimethylsilyl, Mst: 2,4,6-trimethylphenylsulfonyl, TPS: 2,4,6triisopropylsulfonyl.) 
Table 1

Exploration of various activating groups for the reaction of 4-substituted pyrimidin-2-ones with $2 \mathrm{AF}$

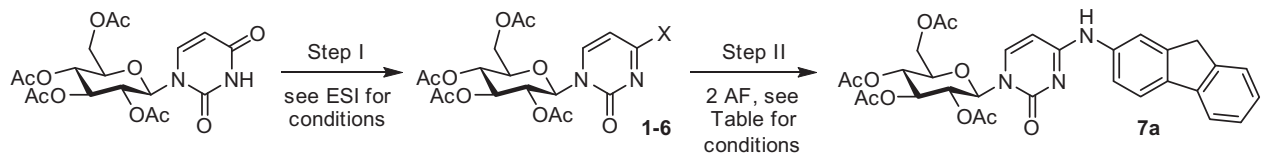

\begin{tabular}{|c|c|c|c|c|c|}
\hline Entry & $\mathrm{X}$ & Conditions for step II ${ }^{a}$ & Temp & Time & Yield \% (over two steps) \\
\hline 1 & $\mathrm{OSiMe}_{3}(\mathbf{1})$ & HMDS & $80^{\circ} \mathrm{C}$ & $10 \mathrm{~h}$ & - \\
\hline 2 & OMst (2) in situ & DCE & $\mathrm{rt}$ & Overnight & $(47)$ \\
\hline 3 & OTPS (3) purified & Dioxane & $70^{\circ} \mathrm{C}$ & $15 \min$ & $85(20)$ \\
\hline 4 & OTPS (3) in situ & DCE & $70^{\circ} \mathrm{C}$ & $2 \mathrm{~h}$ & $(53)$ \\
\hline 5 & Triazole (5) purified & Pyridine & $115^{\circ} \mathrm{C}$ & $10 \mathrm{~h}$ & $55(53)$ \\
\hline 6 & Tetrazole $(\mathbf{6})$ in situ & Pyridine & $50^{\circ} \mathrm{C}$ & $5 \mathrm{~d}$ & $(47)$ \\
\hline
\end{tabular}

a 1.5 equiv of 2 -aminofluorene ( $2 \mathrm{AF})$ was used in all reactions.

arylamines were expected to be less reactive (entry 1 , Table 1 ). We then shifted our attention to the examination of various $O^{4}$-arylsulfonates. ${ }^{14}$ Synthesis of $O^{4}$-mesitylenesulfonyl derivative $\mathbf{2}$ proceeded smoothly in the presence of mesitylenesulfonyl chloride and catalytic DMAP in dichloroethane, however the sulfonated intermediate was not stable enough to be isolated and characterized. Nevertheless, it could be reacted in situ with $2 \mathrm{AF}$ giving the desired product in $47 \%$ yield over 2 steps (entry 2, Table 1 ). Due to the instability of the mesitylenesulfonyl derivative we shifted our attention to the more hindered 2,4,6-triisopropylphenylsulfonyl (TPS) derivative. This was prepared using TPS chloride, triethylamine and catalytic DMAP in dichloroethane ( $\mathrm{rt}, 36 \mathrm{~h}$ ) and was isolated in a modest $23 \%$ yield after chromatography. Quantitative ${ }^{1} \mathrm{H}$ NMR of the crude product, after aqueous workup, indicated the presence of the TPS derivative in 57\% yield. This suggested that the TPS derivative was also relatively unstable to chromatography.

When the isolated TPS derivative 3 (entry 3, Table 1) was reacted with $2 \mathrm{AF}$ in dioxane $\left(70{ }^{\circ} \mathrm{C}, 15 \mathrm{~min}\right)$ the desired product was isolated in high yield (85\%). However, the overall yield for the 2 -step procedure was a modest $20 \%$. In an attempt to further improve the yield, we attempted to react the in situ generated TPS derivative with 2AF. Using this one-pot, two-step procedure, we were able to raise the total yield to $53 \%$ over 2 steps and at the same time simplify the whole process (entry 4, Table 1 ). However, in this case, a small amount of a side product was formed $(<5 \%)$, which was characterized as an $N^{4}, N^{4}$-diethyl cytosine derivative, that had most likely formed from the de-ethylation of a triethylammonium intermediate, since triethylamine remained in the reaction mixture from the initial TPS-formation step.

Following Reese's work ${ }^{2}$ we then attempted to prepare the 4-(3-nitro-1,2,4-triazolyl)pyrimidin-2-one derivative 4, which was expected to be a more stable crystalline intermediate. In the original work, an arabinosyl analogue had been prepared using diphenyl phosphorochloridate and 3-nitro-1,2,4-triazole in pyridine $^{2}$ and more recently with $p$-tosyl chloride, diphenyl phosphate and 3-nitro-1,2,4-triazole. ${ }^{15}$ Under both reaction conditions, we were able to isolate, after chromatographic separation, the previously unreported 3-nitro-1,2,4-triazolyl derivative 4, in microcrystalline form, albeit in low yield (15\%). The low yield and long reaction time ( $72 \mathrm{~h}$ ) required for the formation of 4 meant that this compound was not appropriate as a versatile intermediate for the substitution reaction with arylamines. On the other hand, the 4-(1,2,4-triazolyl)pyrimidin-2-one derivative $\mathbf{5}$ was synthesized using a slight modification of Reese's procedure and was obtained in high yield (95\%) and virtually pure after aqueous workup. It was found that changing the sequence of reagent addition by adding $\mathrm{Et}_{3} \mathrm{~N}$ last, resulted in a faster and cleaner reaction. Compound $\mathbf{5}$ could easily be obtained as a white microcrystalline product after trituration of the off-white crude product with diethyl ether. When triazole derivative $\mathbf{5}$ was reacted with $2 \mathrm{AF}$ in pyridine at reflux, we observed slow conversion to the desired product leading to a $55 \%$ isolated yield after $10 \mathrm{~h}$, without complete consumption of the starting material (entry 5 , Table 1 ).

The last target-intermediate examined in the initial screening was the corresponding tetrazole $\mathbf{6}$, which was prepared utilizing a solution of tetrazole in acetonitrile in the presence of diphenyl phosphate and $p$-tosyl chloride in pyridine. ${ }^{16}$ Under these conditions we observed slow conversion to 6 ( $5 \mathrm{~d}$ ) however the product proved to be unstable upon workup and therefore was reacted in situ with $2 \mathrm{AF}$ at $50^{\circ} \mathrm{C}$. Again we observed slow conversion ( $5 \mathrm{~d}$ ) giving the desired product in $47 \%$ yield after chromatographic separation. It was concluded that the best conditions included either the in situ formation and reaction of TPS derivative 3 or the use of triazole intermediate $\mathbf{5}$. (Table 1, entries 4 and 5, respectively). Nevertheless, since the reaction conditions involving $\mathbf{3}$ had already been optimized, we decided to proceed with optimization of the conditions involving triazolyl-intermediate $\mathbf{5}$, using $2 \mathrm{AF}$ as a model arylamine.

An initial attempt to methylate derivative $\mathbf{5}$ using methyl iodide or dimethyl sulfate ${ }^{17}$ in order to quaternize the triazole and render it a better leaving group was unsuccessful, leading to low yields and long reaction times. Therefore we studied the effect of a number of additives and solvents (Table 2).

The utilization of Hunig's base (entry 1) or DBU (entry 2) in dichloromethane did not improve the yield of the reaction, however the use of DABCO led to a significant increase in yield when

Table 2

Reaction condition optimization for the substitution of 4-(1,2,4-triazolyl)pyrimidin-2one by $2 \mathrm{AF}$

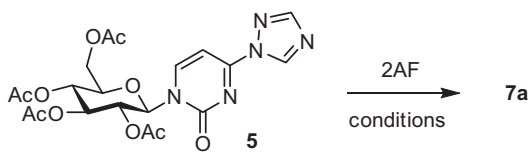

\begin{tabular}{lllll}
\hline Entry & Conditions & Temp & Time & Yield \% \\
\hline 1 & DCM, DIPEA $^{\mathrm{a}}$ & $40{ }^{\circ} \mathrm{C}$ & $72 \mathrm{~h}$ & 55 \\
2 & DCM, DBU $^{\mathrm{a}}$ & $40{ }^{\circ} \mathrm{C}$ & $2 \mathrm{~h}$ & 40 \\
3 & Pyridine, DABCO $^{\mathrm{b}}$ & $115^{\circ} \mathrm{C}$ & $2.5 \mathrm{~h}$ & 74 \\
4 & DCE, DABCO $^{\mathrm{b}}$ & $84{ }^{\circ} \mathrm{C}$ & $2.5 \mathrm{~h}$ & 70 \\
5 & ${\text { Pyridine, } \mathrm{MW}^{\mathrm{b}, \mathrm{c}}}_{6}$ & $80-115^{\circ} \mathrm{C}$ & $0.5 \mathrm{~h}$ & 72 \\
6 & $\mathrm{SiO}_{2}, \mathrm{MW}^{\mathrm{b}, \mathrm{d}}$ & $70{ }^{\circ} \mathrm{C}$ & $10 \mathrm{~min}$ & 90 \\
\hline
\end{tabular}

2.5 equiv of $2 \mathrm{AF}$ was used.

b 1.5 equiv of $2 \mathrm{AF}$ was used.

c The reagents were suspended in pyridine $(0.15 \mathrm{~mL})$, MW power output $150 \mathrm{~W}$.

d $2 \mathrm{~g} \mathrm{SiO}_{2}$ per mmol of $\mathbf{5}$ was added, MW power output $150 \mathrm{~W}$. 
Table 3

MW substitution of the triazolyl group in $\mathbf{5}$ with aryl and alkylamines, using dry $\mathrm{SiO}_{2}$ as an adsorbent ${ }^{22}$

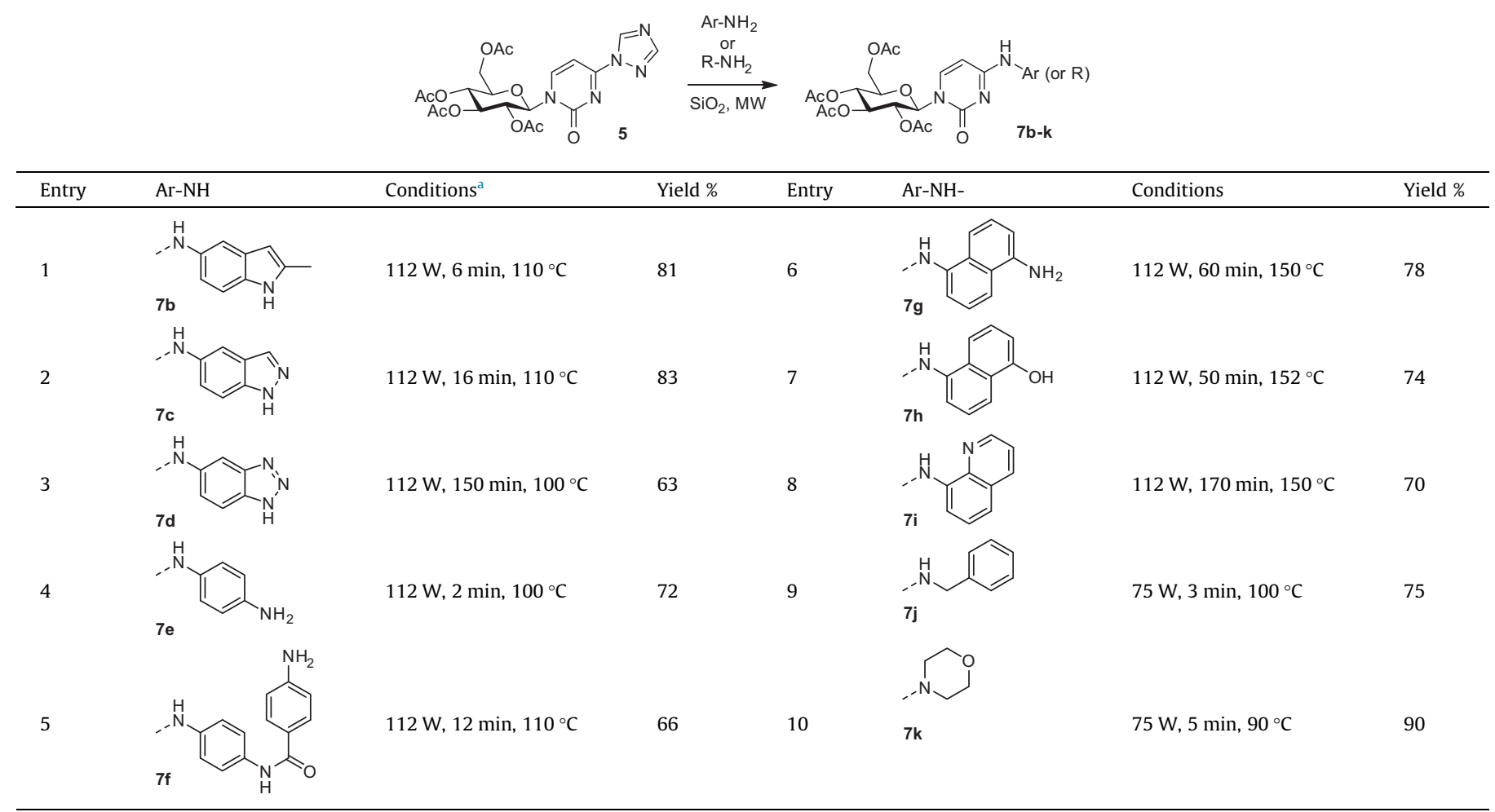

a $2 \mathrm{~g}$ of $\mathrm{SiO}_{2}$ per mmol of $\mathbf{5}$ were used.

the reaction was run in pyridine or 1,2-dichloroethane (entries 3 and 4 , respectively). DABCO has been shown, by us ${ }^{18,19}$ and others, ${ }^{20}$ to activate, via substitution, sulfonates in nucleophilic substitution reactions. The utilization of microwave irradiation is a widely used methodology that can in many cases dramatically improve both yield and reaction times. ${ }^{21}$ In particular, the use of dry media, such as silica gel, in microwave assisted organic synthesis, has provided a means for designing simplified and green synthetic protocols. ${ }^{22}$ Silica gel has widely been used as a dry media adsorbent, since it can be readily combined with product purification by flash column chromatography. When the reaction was performed in pyridine, utilizing a CEM Discover Microwave Synthesis System, the same increase in yield was achieved, as in entry 3, but with a significantly faster reaction time (entry 5 ). When the reactants were adsorbed onto silica gel, the reaction proceeded with an excellent yield (90\%) and had an extremely short reaction time (10 min). Adsorption was achieved by dissolving the reactants in a polar solvent (dichloromethane, acetonitrile or methanol) in the presence of $\mathrm{SiO}_{2}$, and evaporating the solvent until dry, before placing the dry silica in the microwave vessel. This simplified and optimized protocol was subsequently applied to study the reactivity for substitution of triazolyl derivative $\mathbf{5}$ by a number of aryl and alkyl amine substrates (Table 3 ).

Indole derivatives with an increasing number of nitrogen atoms on the 5-membered ring ranging from 5-amino-2-methylindazole to 2-aminobenzotriazole were initially examined (entries 1-3, Table 3). Moving from compounds $\mathbf{7 b}$ to 7d, we observed a remarkable decrease in reactivity, as the number of nitrogen atoms increased, due to their inductive effect, with reaction times increasing from $6 \mathrm{~min}$ to $150 \mathrm{~min} .{ }^{23}$ On the other hand, exocyclic p-aminoaryl groups (entries 4 and 5, Table 3 ) increased the reactivity of the corresponding amine. It should be noted that in the case of entry 5 , a single product was observed, and isolated in $66 \%$ yield.
Due to electronic considerations, the product was assigned as the structure shown in entry 5 , which was further corroborated by crystallographic studies of the fully deprotected derivative in a complex with glycogen phosphorylase. Details of this study will be published elsewhere. Entries 6-8 containing sterically hindered 1 -aminonaphthalene derivatives required increased reaction times (50 min and $60 \mathrm{~min}$ for derivatives $\mathbf{7 g}$ and $\mathbf{7 h}$, respectively) with the slowest reacting derivative being 8 -aminoquinoline (Table 3 , entry 8). Finally, two alkylamine examples were examined, which as expected, reacted readily, providing products $\mathbf{7 j}$ and $\mathbf{7 k}$ in high yields (entries 9 and 10). The limits of the methodology were tested with heterocyclic amines of extremely low nucleophilicity such as adenine or 4-aminophthalimide (results not shown). In these cases the only product observed was the hydrolysis product of derivative 5, $\beta$-D-glucopyranosyluracil, arising from its reaction with silica gel. Traces of $\beta$-D-glucopyranosyluracil $(<5 \%)$ were also observed after prolonged reaction times with the most unreactive arylamines (entries 3 and 7 ). Attempts to eliminate this side product by prolonged drying of the silica gel (heated at $60{ }^{\circ} \mathrm{C}$ under vacuum for $3 \mathrm{~h}$ ) prior to use, or by utilizing $\mathrm{C}-18$ reverse phase silica gel were unsuccessful. It appeared that the formation of $\beta$-D-glucopyranosyluracil was inversely proportional to the reactivity of the corresponding arylamine and it was assumed that this product arose from nucleophilic addition of the silica gel surface silanols to the triazole derivative, followed by hydrolysis upon final purification.

\section{Conclusions}

The reported methodology corresponds to a robust procedure for the synthesis of a variety of $\beta$-D-glucopyranosyluracil derivatives in good to excellent yields. The reaction times for the 
reported examples were fast, under the microwave conditions employed, but varied depending on the electronic and stereochemical factors which controlled the reactivity of the corresponding amines. With less reactive amines and longer reaction times, the formation of small amounts $(<5 \%)$ of $\beta$-D-glucopyranosyluracil was observed and became prevalent when unreactive heterocyclic amines were utilized. One of the strengths of the current method is that it does not require aqueous workup, and direct purification of the final product can be achieved by directly loading the reaction mixture onto a column, which minimizes loss of material and simplifies the whole synthetic procedure. The current methodology extends the use of solvent free, benign media in microwavemediated reactions ${ }^{14}$ and may find wider applications in nucleoside chemistry. The biological activity of some of the newly reported products as inhibitors of glycogen phosphorylase will be reported in due course.

\section{Acknowledgments}

This research has been co-financed by the European Union (European Social Fund-ESF) and Greek national funds through the Operational Program 'Education and Lifelong Learning' of the National Strategic Reference Framework (NSRF)-Research Funding Program: Heracleitus II. Investing in knowledge society through the European Social Fund. The support and sponsorship of the COST framework program, Action CM1201, on Biomimetic Radical Chemistry, is gratefully acknowledged.

\section{Supplementary data}

Supplementary data associated with this article can be found, in the online version, at http://dx.doi.org/10.1016/j.tetlet.2015.08. 037.

\section{References and notes}

1. Reese, C. B. Tetrahedron 2002, 58, 8893-8920.

2. Divakar, K. J.; Reese, C. B. J. Chem. Soc., Perkin Trans. 1 1982, 1171-1176.

3. Medvecky, M.; Istrate, A.; Leumann, C. J. J. Org. Chem. 2015, 80, 3556-3565.

4. Jonkers, T. H. M.; Lin, T.-I.; Buyck, C.; Lachau-Durand, S.; Vandyck, K.; Van Hoof, S.; Vandekerckhove, L. A. M.; Hu, L.; Berke, J. M.; Vijgen, L.; Dillen, L. L. A. Cummings, M. D.; de Kock, H.; Nilsson, M.; Sund, C.; Rydegard, C.; Samuelsson, B.; Rosenquist, A.; Fanning, G.; Van Emelen, K.; Simmen, K.; Raboisson, P. J. Med. Chem. 2010, 53, 8150-8160.

5. Peng, X.; Hong, I. S.; Li, H.; Seidman, M. M.; Greenberg, M. M. J. Am. Chem. Soc 2008, 130, 10299-10306.

6. Cismas, C.: Gimisis, T. Tetrahedron Lett. 2008, 49, 1336-1339.

7. Noll, D. M.; Noronha, A. M.; Miller, P. S. J. Am. Chem. Soc. 2001, 123, 3405-3411.

8. Plitta, B.; Adamska, E.; Giel-Pietraszuk, M.; Fedoruk-Wyszomirska, A.; NaskrętBarciszewska, M.; Markiewicz, W. T.; Barciszewski, J. Eur. J. Med. Chem. 2012, $55,243-254$.

9. Schott, H.; Goltz, D.; Schott, T. C.; Jauch, C.; Schwendener, R. A. Bioorg. Med. Chem. 2011, 19, 3520-3526.

10. Moller, D. E. Nature 2001, 414, 821-827.

11. Gimisis, T. Mini-Rev. Med. Chem. 2010, 10, 1127-1138.

12. Chrysina, E. D. Mini-Rev. Med. Chem. 2010, 10, 1093-1101.

13. Vorbrueggen, H. Acc. Chem. Res. 1995, 28, 509-520.

14. Bischofberger, N. Tetrahedron Lett. 1987, 28, 2821-2824.

15. Lagoja, I. M.; Pochet, S.; Boudou, V.; Little, R.; Lescrinier, E.; Rozenski, J.; Herdewijn, P. J. Org. Chem. 2003, 68, 1867-1871.

16. Ariza, X.; Vilarrasa, J. J. Org. Chem. 2000, 65, 2827-2829.

17. Ermakova, T. G.; Tatarova, L. A.; Gritsa, A. I.; Kuznetsova, N. P.; Chipanina, N. N.; Lopyrev, V. A. Chem. Heterocycl. Compd. 1984, 20, 1167-1169.

18. Vrantza, D.; Kaloudis, P.; Leondiadis, L.; Gimisis, T.; Vougioukalakis, G. C.; Orfanopoulos, M.; Gasparutto, D.; Cadet, J.; Encinas, S.; Paris, C.; Miranda, M. A. Helv. Chim. Acta 2006, 89, 2371-2386.

19. Kaloudis, P.; Paris, C.; Vrantza, D.; Encinas, S.; Perez-Ruiz, R.; Miranda, M. A.; Gimisis, T. Org. Biomol. Chem. 2009, 7, 4965-4972.

20. Lakshman, M. K.; Ngassa, F. N.; Keeler, J. C.; Dinh, Y. Q. V.; Hilmer, J. H.; Russon, L. M. Org. Lett. 2000, 2, 927-930.

21. Polshettiwar, V.; Varma, R. S. Acc. Chem. Res. 2008, 41, 629-639.

22. Gawande, M. B.; Shelke, S. N.; Zboril, R.; Varma, R. S. Acc. Chem. Res. 2014, 47 1338-1348.

23. Katritzky, A. F.; Pozharski, A. F. Handbook of Heterocyclic Chemistry, 2nd ed.; Pergamon Press, 2000. pp 171, 377-379. 